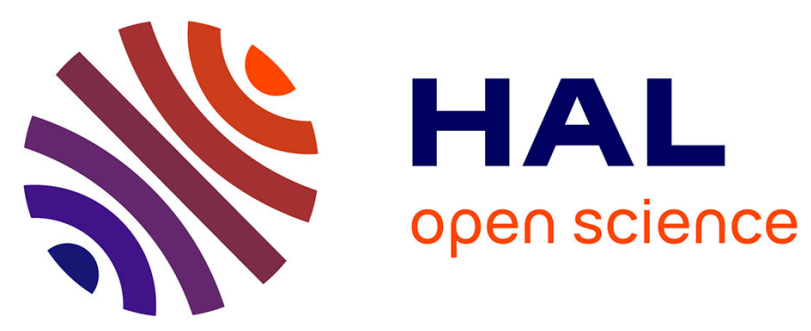

\title{
Pourquoi les inégalités de patrimoine sont-elles mieux tolérées que d'autres?
}

Michel Forse, Alexandra Frenod, Maxime Parodi, Caroline Guibet Lafaye

\section{To cite this version:}

Michel Forse, Alexandra Frenod, Maxime Parodi, Caroline Guibet Lafaye. Pourquoi les inégalités de patrimoine sont-elles mieux tolérées que d'autres?. Revue de l'OFCE, 2018, Aspects des transmissions familiales entre générations, 156, pp.97-122. 10.3917/reof.156.0097 . hal-03458231

\section{HAL Id: hal-03458231 \\ https://hal-sciencespo.archives-ouvertes.fr/hal-03458231}

Submitted on 30 Nov 2021

HAL is a multi-disciplinary open access archive for the deposit and dissemination of scientific research documents, whether they are published or not. The documents may come from teaching and research institutions in France or abroad, or from public or private research centers.
L'archive ouverte pluridisciplinaire HAL, est destinée au dépôt et à la diffusion de documents scientifiques de niveau recherche, publiés ou non, émanant des établissements d'enseignement et de recherche français ou étrangers, des laboratoires publics ou privés.

\section{(ㅇ)(1) $\$$}

Distributed under a Creative Commons Attribution - NonCommercial - NoDerivatives 44.0 


\title{
POURQUOI LES INÉGALITÉS DE PATRIMOINE SONT-ELLES MIEUX TOLÉRÉES QUE D'AUTRES?
}

\author{
Michel Forsé \\ CNRS, CMH \\ Alexandra Frénod \\ CNRS, GEMASS \\ Caroline Guibet Lafaye \\ CNRS, Centre Emile Durkheim \\ Maxime Parodi \\ Sciences Po, OFCE
}

Les sondages, et notamment celui qui est étudié dans cet article, se succèdent pour montrer que les Français sont plus tolérants à l'égard des inégalités de patrimoine que vis-à-vis d'autres types d'inégalités, même lorsqu'elles sont aussi à caractère économique. Une enquête par entretiens semi-directifs auprès de trois générations de 35 lignées familiales $(n=105)$ a permis de mettre à jour trois logiques propres venant structurer les opinions sur la transmission patrimoniale : celle du libre agent, celle de l'égalité citoyenne et celle que l'on peut qualifier de familialiste. Quelle que soit cette logique, beaucoup d'interviewés soulignent aussi l'importance de la transmission culturelle et/ou affective. II faut d'ailleurs noter que les membres d'une même lignée ont tendance à partager des opinions assez proches. Pour les niveaux plutôt faibles de patrimoine auxquels ils songent spontanément, ils manifestent une très forte aversion face à l'idée de taxer l'héritage, surtout s'il s'agit de la maison familiale. Pour des niveaux beaucoup plus élevés, une taxation importante n'est cette fois guère contestée.

Mots clés : inégalités, patrimoine, héritage, famille, reproduction sociale, générations, opinions, justice sociale. 
«Le droit de succession ex testamento est l'une des plus grandes extensions concevables du droit de propriété »(Smith, 1763, p. 92/51).

«...le droit de propriété [...] ne s'étend point au-delà de la vie du propriétaire, et [...] à l'instant qu'un homme est mort, son bien ne lui appartient plus. Ainsi lui prescrire les conditions sous lesquelles il en peut disposer, c'est au fond moins altérer son droit en apparence, que l'étendre en effet » (Rousseau, 1755, p. 26)

\section{E} ntre les formules de ces deux philosophes du XVIII ${ }^{\mathrm{e}}$ siècle, le cœur des Français balance peu : ils sont largement smithiens. Pourtant tel n'est pas là le moindre des paradoxes que les Français nourrissent et ce, pour deux raisons. D'une part, comme nous le verrons de façon approfondie, ils témoignent d'une aversion remarquable aux inégalités. D'autre part, ils sont, dans leur grande majorité, ignorants des règles de succession dans leur propre pays. En effet, lorsque l'institut de sondage BVA les interrogent sur la possibilité de déshériter entièrement I'un de ses enfants ou de déshériter entièrement un ascendant ainsi que sur la nécessité de faire cosigner par ceux qui en bénéficieront son testament, seuls $13 \%$ des personnes interrogées donnent une bonne réponse à chacune de ces questions (BVA, « Les Français et l'héritage ", 2011). Plus encore, tous ( $99 \%$ pour être exact) se sont trompés sur au moins une des questions portant sur les ordres de grandeur des taux de taxation exigés lors d'une succession entre conjoints mariés, ou entre conjoints pacsés ou encore entre concubins. Et la majorité des personnes interrogées ignore même l'ordre de grandeur de la taxation pour le cas les concernant. Ainsi seules $8 \%$ des personnes en unionlibre savent qu'elles seront taxées à $60 \%$ si leur conjoint décède (et $30 \%$ d'entre elles pensent même que cette taxation est inférieure à $5 \%)^{1}$.

Au-delà de ce manque de connaissances juridiques, les attitudes des Français à l'égard des droits de succession et de l'héritage sont ancrées dans des représentations et une perception des inégalités dichotomiques, celles-ci pouvant être interprétées, par certains, comme prédéterminées et dues à des déterminismes sociaux, alors que d'autres jugeront qu'elles sont essentiellement causées par des facteurs sur lesquels l'individu a prise (tels l'effort individuel, l'ambition, la

1. Cette méconnaissance est confirmée par un sondage récent du CREDOC (juin $2017, \mathrm{n}=2004$ ) sur la fiscalité des transmissions patrimoniales (Grégoire-Marchand, 2018). 
persévérance, la pugnacité, le sacrifice, etc.). Mais, au-delà, ces représentations se transmettent-elles d'une génération à une autre? Certains phénomènes, comme les préférences politiques, se «transmettent » (ou se transmettaient) de manière transgénérationnelle (voir Percheron, 1982, 1993). On retrouve plus ou moins cette transmission s'agissant des choix d'épargne (Arrondel, 2009). En effet, nous avons là affaire à des processus d'apprentissage rationnels, où chacun utilise au mieux l'information à laquelle il a été naturellement exposé, au cours de son expérience personnelle, en particulier familiale. Si l'on passe aux questions touchant l'héritage, en va-t-il de même?

Afin de comprendre la genèse et l'éventuelle transmission/reproduction des mécanismes, conduisant les individus à endosser telles ou telles représentations, voire telles ou telles croyances, en matière de patrimoine, nous avons mené, entre avril 2014 et octobre 2015, une enquête qualitative transgénérationnelle. Les entretiens semi-directifs conduits auprès de 105 personnes, appartenant à 35 lignées, visaient à préciser le poids de l'origine sociale et des croyances familiales (en matière de genèse des inégalités), dans l'affirmation de convictions morales et de préférences idéologiques entre les générations ${ }^{2}$. Le premier objectif était d'identifier une éventuelle stabilité de ces convictions et préférences d'une génération à l'autre et de préciser les mécanismes de leur transmission au sein de la famille. Le second objectif consistait à se demander si les opinions sur la transmission patrimoniale et les inégalités qui l'accompagnent relevaient d'une logique propre ou au contraire ne faisaient que prolonger ce que l'on observe pour d'autres domaines économiques comme les revenus.

Du côté objectif, il faut en effet rappeler que les inégalités de patrimoine brut (sans déduction des dettes) des ménages restent importantes même si, selon I'INSEE (Ferrante, Guillas et Solotareff, 2016), elles ont baissé très légèrement entre 2010 et 2015. Les indica-

2. Ces entretiens ont été menés dans le cadre du programme « DYNEGAL " (cf. le site dynegal.org pour plus de détails) financé par l'Agence Nationale de la Recherche. D'une durée moyenne de 45 minutes, ils ont permis d'interroger 3 générations d'individus d'une même lignée ( $1 / 3$ de 15 25 ans, $1 / 3$ de $26-60$ ans $1 / 3$ de plus de 60 ans). Ils ont porté sur 41 hommes et 64 femmes établis, en milieu rural, périurbain ou urbain, dans différentes régions françaises (Paris, lle-de-France, Hautsde-France, Grand-Est, Provence-Alpes-Côte-d'Azur, Occitanie, Nouvelle-Aquitaine, Pays-de-la-Loire, Bretagne, Guadeloupe). Une attention particulière a été portée à la variété des origines religieuses et sociodémographiques (regroupés en grandes catégories, toutes les situations de ménages, tous les niveaux d'études, tous les groupes socioprofessionnels, toutes les fourchettes de revenus, toutes les situations de logements, toutes les positions de l'échelle politique se sont rencontrées au moins une fois). 
teurs statistiques d'inégalité convergent à l'appui de ce diagnostic. Par exemple, l'indice de Gini (qui varie entre 0 pour une parfaite égalité et 1 pour le maximum d'inégalité envisageable) vaut, début 2015, 0,653 contre 0,662 , début 2010 . À ces deux dates, les biens immobiliers forment la part la plus importante $(61 \%)$ du patrimoine brut des ménages et, parmi ceux qui possèdent de tels biens, la résidence principale en constitue, de loin, la composante essentielle (en l'occurrence, $85 \%$ de la valeur des biens immobiliers détenus par ces ménages). De fait, en 2015, environ $60 \%$ des ménages sont propriétaires ou accèdent à la propriété de leur résidence principale. Mais pour revenir aux inégalités, seuls $25 \%$ des ménages, dont le patrimoine brut est inférieur au patrimoine médian, sont dans ce cas, contre $95 \%$ de ceux dont le patrimoine brut se situe dans le dernier décile (celui des $10 \%$ les plus riches). Au total, le patrimoine brut des propriétaires ou accédants à la propriété de leur résidence principale est en valeur à peu près sept fois et demie plus élevé que celui des locataires ou des personnes logées à titre gratuit.

Cette concentration du patrimoine, même si elle repose majoritairement sur la propriété de la résidence principale, est bien plus forte que celle qui se rencontre dans d'autres domaines économiques touchant à la richesse. Ainsi, même si les inégalités de revenus ne sont pas négligeables, elles sont loin d'atteindre ce niveau. Selon I'INSEE, au-delà de variations de court terme (Argouarc'h et Boiron, 2016), l'indice de Gini se situe au cours des dernières années aux alentours de 0,3 , soit une valeur plus que deux fois moins forte que celle que l'on vient de rappeler pour le patrimoine brut des ménages.

Peut-on en déduire que les Français jugent plus sévèrement les inégalités de patrimoine que d'autres types d'inégalités, notamment de revenus ? Formulent-ils des critiques à l'encontre des héritages ou des donations qui sont, pour une bonne part, à la source de l'inégale concentration des richesses ? Cela ne serait pas très étonnant puisqu'ils se distinguent par une forte " passion de l'égalité » par comparaison avec ce que l'on observe dans d'autres pays (Forsé, 2013; Forsé et al., 2013).

Les enquêtes par sondage que nous allons examiner ici vont pourtant montrer que ce n'est pas le cas. Dès lors il va s'agir, au travers de l'analyse des entretiens semi-directifs évoqués plus haut, de comprendre les raisons de ce paradoxe. Pourquoi les Français, qui 
dénoncent par ailleurs fortement les inégalités, sont-ils plus tolérants lorsqu'il s'agit du patrimoine et de sa transmission ?

Pour trouver des éléments de réponse, nous commencerons par examiner les opinions sur les inégalités de patrimoines et sur l'équité des règles de succession à partir d'une enquête représentative de la population française, puis nous approfondirons cet examen par une étude plus qualitative sur les raisons qui rendent plus ou moins acceptables ces inégalités aux yeux de nombreux Français.

\section{Des inégalités de patrimoine jugées moins fortes et plus acceptables que d'autres inégalités économiques}

Pour établir le diagnostic s'agissant des attitudes des Français dans leur ensemble, l'enquête «Perceptions des Inégalités et Sentiments de Justice » (PISI) ${ }^{3}$ (Forsé et Galland, 2011 ; Forsé et al., 2013) s'avère d'une aide précieuse car elle permet, pour une série de 12 types d'inégalités, de s'interroger simultanément sur la perception de leur intensité, sur les opinions relative à leur acceptabilité et sur la position personnelle de l'enquêté à leur égard. Les questions posées et les résultats obtenus sont détaillés dans les tableaux 1,2 et 3 . Les inégalités économiques (qui, dans l'enquête, renvoyaient aux revenus, à la pénibilité du travail, au logement, à l'emploi et au patrimoine) y apparaissent les plus fortes, davantage notamment que celles à caractère plus identitaire (sexe, âge, origine ethnique) ou autre. En revanche, elles semblent plus acceptables que ces dernières. Cependant, parmi ces inégalités économiques, les inégalités de patrimoine sont considérées comme les moins intenses. Elles sont d'ailleurs jugées comme étant les plus acceptables de toutes, bien que les enquêtés se disent davantage défavorisés dans ce domaine que dans d'autres (à l'exception de ce qui a trait aux écarts de revenus).

3. Cette enquête a été réalisée par I'Institut de Sondage Lavialle (GfK-ISL) en face à face au domicile de l'enquêté (grâce à un financement accordé par la Fondation Simone et Cino Del Duca) en septembre et octobre 2009 auprès d'un échantillon de 1711 individus représentatifs par quota de la population de 18 ans et plus résidant en France métropolitaine. Le plan de sondage a été calculé sur une matrice croisée détaillant les 9 régions ZEAT et la catégorie d'agglomération ventilée en 9 classes. Une pondération a été calculée a posteriori pour redresser selon le sexe, l'âge et la profession et a été utilisée dans cet article. Mais, comme les consignes données aux enquêteurs, et qui sont destinées à caler la composition de l'échantillon sur ce que sont les proportions effectives des différentes catégories démographiques ou professionnelles en France, ont été particulièrement bien suivies, l'effet de ce redressement est extrêmement modeste. En d'autres termes, tenir compte ou non du redressement n'affecte que marginalement un résultat donné et ne change en tous cas pas la conclusion que l'on peut en tirer. Cette même remarque vaut pour le sondage «Dynegal » qui sera utilisé un peu plus loin dans cet article. 
Tableau 1. Il existe plusieurs types d'inégalités dans la société française.

Pour celles que je vais vous citer, dites-moi si selon vous elles sont fortes ou pas aujourd'hui ? Donnez votre réponse sur une échelle allant de 1 pour « Pas fortes du tout »

à 10 pour «Très fortes »

Moyennes sur 10

les inégalités de revenu

7,87

les inégalités de pénibilité du travail

7,44

les inégalités de logement

7,41

les inégalités face au chômage et aux emplois précaires

7,36

les inégalités de patrimoine

7,20

les inégalités liées à l'origine ethnique

6,90

les inégalités dans les études scolaires

6,55

les inégalités entre les hommes et les femmes

6,55

les inégalités face à l'insécurité

6,51

les inégalités d'accès aux soins médicaux

6,30

les inégalités entre les jeunes et les plus âgés

6,30

les inégalités d'exposition aux risques technologiques, industriels ou scientifiques (nucléaire, OGM, santé)

6,15

Tableau 2. Pour chacune de ces inégalités, quelles sont celles qui vous semblent plus ou moins acceptables?

Donnez chaque fois votre réponse sur une échelle allant de 1 pour «ne sont jamais acceptables » à 10 pour « sont toujours acceptables »

Moyennes sur 10

les inégalités de patrimoine

les inégalités de revenu

les inégalités d'exposition aux risques technologiques, industriels

ou scientifiques (nucléaire, OGM, santé)

les inégalités dans les études scolaires

3,62

les inégalités entre les jeunes et les plus âgés

les inégalités face au chômage et aux emplois précaires

les inégalités face à l'insécurité

les inégalités liées à l'origine ethnique

les inégalités de logement

3,22

les inégalités entre les hommes et les femmes

3,14

les inégalités d'accès aux soins médicaux 
Tableau 3. Dans chacun des domaines suivants où peuvent exister des inégalités, vous sentez-vous vous-même en position très défavorisée, assez défavorisée, plutôt favorisée ou très favorisée?

\begin{tabular}{lrrcc} 
& $\begin{array}{c}\text { Très défa- } \\
\text { vorisée }\end{array}$ & $\begin{array}{c}\text { Assez défa- } \\
\text { vorisée }\end{array}$ & $\begin{array}{c}\text { Plutôt } \\
\text { favorisée }\end{array}$ & $\begin{array}{c}\text { Très } \\
\text { favorisée }\end{array}$ \\
\hline en matière de revenu & $13 \%$ & $38 \%$ & $46 \%$ & $3 \%$ \\
en matière de patrimoine & $13 \%$ & $31 \%$ & $51 \%$ & $5 \%$ \\
en matière de pénibilité du travail & $7 \%$ & $28 \%$ & $51 \%$ & $14 \%$ \\
$\begin{array}{l}\text { en matière de chômage et d'emplois précaires } \\
\text { en matière d'études scolaires }\end{array}$ & $10 \%$ & $24 \%$ & $48 \%$ & $18 \%$ \\
$\begin{array}{l}\text { en matière d'inégalités entre les hommes et } \\
\text { les femmes }\end{array}$ & $6 \%$ & $22 \%$ & $62 \%$ & $10 \%$ \\
$\begin{array}{l}\text { en matière d'exposition aux risques technolo- } \\
\text { giques, industriels ou scientifiques (nucléaire, }\end{array}$ & $5 \%$ & $19 \%$ & $57 \%$ & $19 \%$ \\
$\begin{array}{l}\text { OGM, santé, etc.) } \\
\text { en matière d'inégalités entre les jeunes et }\end{array}$ & $3 \%$ & $21 \%$ & $67 \%$ & $9 \%$ \\
$\begin{array}{l}\text { les plus âgés } \\
\text { en matière d'insécurité }\end{array}$ & $5 \%$ & $16 \%$ & $65 \%$ & $14 \%$ \\
en matière de logement & $5 \%$ & $15 \%$ & $63 \%$ & $17 \%$ \\
en matière d'accès aux soins médicaux & $4 \%$ & $15 \%$ & $66 \%$ & $15 \%$ \\
$\begin{array}{l}\text { en matière d'inégalités liées à l'origine } \\
\text { ethnique }\end{array}$ & $4 \%$ & $12 \%$ & $55 \%$ & $29 \%$ \\
\hline
\end{tabular}

En résumé, les inégalités de patrimoine sont celles qui, dans le domaine économique, sont jugées les moins fortes et les plus acceptables ${ }^{4}$, bien que chacun se considère ici davantage défavorisé que face à d'autres types d'inégalités - les inégalités de revenu faisant exception. Ainsi plus on se juge défavorisé en matière d'inégalités économiques, plus on aura tendance à les trouver fortes. Ceux qui pensent que ces inégalités ne sont jamais acceptables ont davantage tendance que la moyenne à dire qu'elles sont élevées, contrairement à ceux qui les jugent toujours acceptables et qui affirment moins que la moyenne que ces inégalités sont importantes dans la société française.

Ces jugements sur l'intensité des inégalités de patrimoine ne sont que faiblement expliqués par les postions sociodémographiques ou économiques usuelles ${ }^{5}$. Les sentiments de justice ou d'injustice, que ce soit en ce qui concerne la société française dans son ensemble ou son propre revenu, n'ont pas non plus d'effet sur les jugements en matière

4. On trouve un résultat identique dans les baromètres de la DREES (sondage représentatif réalisé par BVA auprès d'environ 3000 individus) de 2015 ou 2016 où ici aussi, parmi huit types d'inégalités proposées à l'enquêté, celles touchant à l'héritage familial sont jugées à la fois les moins répandues et les moins inacceptables. 
$d^{\prime}$ inégalité de patrimoine. Pour le dire autrement, la façon dont on juge la répartition du patrimoine entre les Français n'est pas à la source d'un sentiment de justice ou d'injustice sociale.

C'est finalement l'opinion politique (auto-déclarée sur une échelle de 1, pour l'extrême-gauche à 10 pour l'extrême-droite mais regroupée ci-après en cinq catégories) qui s'avère avoir ici le plus d'impact (significatif au seuil de 1 pour 10000 dans une analyse de variance). Ceux qui se positionnent à gauche trouvent, plus que la moyenne, que les inégalités de patrimoine sont fortes (au seuil de $1 \%$ dans cette ANOVA). Les individus qui se positionnent au centre de l'échiquier politique n'ont pas un sentiment significativement différent de la moyenne (au seuil de $10 \%$ ). Et ceux qui déclarent avoir des préférences politiques pour la droite pensent qu'il y a moins d'inégalités, dans ce domaine, que la moyenne (au seuil de $1 \%$ ). À gauche, comme à droite, ces tendances sont d'autant plus marquées que l'on va vers les extrêmes (cf. tableau 4).

Tableau 4. Les jugements sur l'intensité des inégalités de patrimoine (sur une échelle de 1 à 10) selon l'opinion politique

\begin{tabular}{|c|c|c|c|}
\hline \multirow[b]{2}{*}{ Opinions politiques } & \multicolumn{3}{|c|}{ Inégalités de patrimoine } \\
\hline & Moyenne & Écart-type & Effectif \\
\hline Extrême-gauche & $7,76^{*}$ & 2,207 & 196 \\
\hline Gauche & 7,55 * & 1,978 & 456 \\
\hline Centre & 7,02 & 2,121 & 475 \\
\hline Droite & 6,81 * & 2,002 & 338 \\
\hline Extrême-droite & 6,62 * & 2,311 & 61 \\
\hline Total & 7,21 & 2,102 & 1526 \\
\hline
\end{tabular}

* = écart à la moyenne significatif au seuil de $1 \%$ dans une ANOVA.

5. Le sexe n'a pas d'influence. L'âge n'intervient que pour les 30 à 59 ans qui trouvent ces inégalités plus fortes que la moyenne. Parmi les groupes socioprofessionnels, seuls les cadres et les catégories intermédiaires ont un jugement plus tranché, estimant davantage que les inégalités de patrimoine sont fortes. II en va de même de ceux qui ont atteint un deuxième ou troisième cycle universitaire, tandis que les diplômés de l'enseignement professionnel (avant le supérieur) ou ceux qui n'ont pas dépassé un niveau d'études primaire ont tendance à dire que les inégalités de patrimoine sont moins fortes que ne le disent les autres. Le niveau de revenu personnel de l'enquêté intervient également peu. Ceux qui gagnent entre 800 et 1200 euros par mois ont tendance à moins dire que les inégalités de patrimoine sont élevées, à l'inverse de ceux qui gagnent entre 1700 et 2400 euros qui les voient plus fortes qu'en moyenne. Contrairement à ce que l'on pourrait penser, le fait d'être propriétaire, accédant ou locataire ne conduit pas à juger qu'il y a plus ou moins d'inégalité de patrimoine. 
Au total, le dissensus sur l'intensité des inégalités de patrimoine tient davantage à l'opinion politique que l'on soutient qu'à la position économique que l'on occupe (aux quelques exceptions près signalées). Dans le même temps, comme nous l'avons vu (cf. tableau 2), cette inégalité est considérée comme la plus acceptable parmi les douze étudiées dans l'enquête. Or en ce qui concerne cette acceptabilité, le diagnostic n'est pas tout à fait le même que celui qui vient d'être posé à propos de l'intensité. En la matière, les positions socio-économiques jouent un rôle explicatif plus important.

Certes, le sexe et l'âge continuent de ne pas avoir de réel impact, tout comme le fait d'être propriétaire, locataire ou accédant à la propriété. En revanche, la catégorie socioprofessionnelle a un effet significatif $(F=4,76, d d l=6, p<0,001$, dans une ANOVA). Cet effet est d'ailleurs surtout dû aux cadres et professions intellectuelles ou libérales $(5,35)$ qui considèrent bien plus que la moyenne $(4,81)$ que les inégalités de patrimoine sont acceptables, contrairement aux ouvriers, employés et femmes au foyer $(4,53)$.

Cette opposition entre le haut et le bas de la hiérarchie des professions est corroborée par ce que l'on observe pour des variables qui y sont corrélées comme le niveau d'études et les revenus. Plus le diplôme est élevé ou plus les revenus sont importants, plus l'acceptabilité est forte. Du coup, si ceux qui s'estiment défavorisés (et qui sont bien ceux qui sont en bas des hiérarchies) trouvent l'inégale répartition des patrimoines moins acceptable, ceci les conduit à un sentiment d'injustice, que ce soit pour la société française prise globalement ou pour leur propre rémunération.

Cela n'empêche pas I'opinion politique de jouer encore un rôle. II est d'ailleurs très simple à résumer : plus on se positionne à droite, plus on juge que les inégalités de patrimoine sont acceptables. Mais souvenons-nous que l'on avait aussi tendance en ce cas à les considérer moins fortes.

Si l'explication de l'acceptabilité des inégalités de patrimoine diffère de celle de leur intensité perçue, ce n'est pas tant en raison du rôle des opinions politiques qui ont dans les deux cas un impact important et congruent, qu'en raison du faible effet de la position socio-économique s'agissant de l'intensité mais qui introduit un clivage entre le haut et le bas de la hiérarchie sociale s'agissant de l'acceptabilité. II n'en demeure pas moins que nous ne jugeons ici que des écarts (significatifs) à la moyenne des réponses. Or quelles que soient les catégories 
sociales ou les opinions politiques, les moyennes les concernant indiquent toujours une acceptabilité plus grande de l'inégalité de patrimoine relativement aux autres types d'inégalités étudiées, tout comme elles vont toujours dans le sens de la perception d'une forte intensité mais moindre que s'agissant des autres types d'inégalités économiques abordées dans l'enquête, en particulier concernant les revenus.

Pour le dire autrement, les hiérarchies observées jusqu'ici ne sont pas bouleversées, lorsque les opinions s'écartent de la moyenne. Ainsi les ouvriers ou employés jugent les inégalités de patrimoine moins acceptables que les autres catégories socioprofessionnelles. Néanmoins, parmi ces catégories populaires, la hiérarchie de l'acceptabilité des différents types d'inégalités est la même que dans l'ensemble de l'échantillon. En particulier, I'inégalité de patrimoine reste la plus tolérable, devant celle touchant aux revenus, tandis que celle concernant l'accès aux soins médicaux reste la plus inacceptable. Il en va de même pour la gauche radicale. Et s'agissant de cette dernière, on observe également qu'elle continue d'attribuer le même rang que la moyenne de la population à l'intensité de l'inégalité de patrimoine vis-à-vis des autres types d'inégalités évoqués.

Force est de constater que, sans se leurrer sur l'inégale répartition des patrimoines, les Français sont ici relativement cléments dans leurs jugements. Bien que les inégalités de revenus soient objectivement moins importantes que celles touchant aux patrimoines, ces dernières sont jugées moins fortes et plus tolérables. Et ceci fait l'objet d'un relatif consensus.

Le patrimoine qui s'acquiert par le travail grâce à une épargne et des emprunts ne pose sans doute pas de difficulté aux Français. II semblerait qu'un jugement comparable affecte le patrimoine transmis par héritage ou donation. Certes I'Impôt de Solidarité sur la Fortune (ISF) ne fait pas de distinction quant au mode d'acquisition et les Français en approuvent majoritairement le principe. Dans le sondage «Dynegal " ${ }^{6}$ (Galland, 2016), il n'est considéré comme un impôt injuste que par environ $20 \%$ d'entre eux. Or l'enquête PISJ avait révélé un consensus sur le principe de la donation, même si ce consensus diminuait lorsque les sommes en jeu augmentaient. C'est ce que

6. Cette enquête a été réalisée par entretiens en face à face en 2013 par TNS-SOFRES (devenu récemment TNS-KANTAR) sur un échantillon représentatif par quota de 4049 adultes résidant en France métropolitaine ( $c f$. le site dynegal.org pour plus de détails). 
montrent les réponses aux deux questions reproduites au tableau $5 \mathrm{a}$ et $b$. L'enquête Dynegal permet quant à elle de constater que les Français ( $84 \%$ d'entre eux) trouvent qu'il y a trop d'impôts et de taxes en France et qu'eux-mêmes en payent trop (73\% sont d'accord avec cette idée); ces opinions étant d'ailleurs d'autant plus affirmées que I'on est pauvre (Forsé et Parodi, 2015).

Tableau 5. M. Martin est un retraité très aisé. Il fait un don de $20000 €$ à sa petite-fille. Vous vous dites plutôt :

\begin{tabular}{ll}
\hline C'est très bien, ça l'aidera à démarrer dans la vie & $84 \%$ \\
\hline Tout le monde n'a pas cette chance, ça crée encore plus d'inégalités sociales & $16 \%$ \\
\hline
\end{tabular}

Tableau 6. Et si I'on vous disait qu'il fait un don à sa petite fille non pas de $20000 €$ mais de $200000 €$. Vous vous diriez plutôt?

\begin{tabular}{ll}
\hline C'est très bien, ça l'aidera à démarrer dans la vie & $54 \%$ \\
\hline Tout le monde n'a pas cette chance, ça crée encore plus d'inégalités sociales & $46 \%$ \\
\hline
\end{tabular}

Or, de ce point de vue, les Français sont particulièrement critiques à l'égard de la fiscalité sur l'héritage et ils seraient en tous cas très réticents à l'alourdir. Quand on leur expose le scénario, « supposons qu'il y ait, au moment d'un héritage, une taxation de 100000 euros sur un patrimoine composé uniquement d'actions cotées en bourse d'un montant de 500000 euros (soit un taux d'imposition de $20 \%$ ) », ils sont $51 \%$ à répondre (parmi ceux qui ont une opinion, soit $93 \%$ de l'échantillon) que cela leur paraît « trop », $40 \%$ « juste ce qu'il faut » et seulement $9 \%$ « pas assez ». Et lorsqu'on expose ce même scénario en y remplaçant simplement dans sa formulation "actions cotées en bourse » par «maison familiale », la désapprobation de la taxation de I'héritage se fait encore plus nette. Ils sont cette fois $77 \%$ à dire que cela leur paraît «trop», $21 \%$ «juste ce qu'il faut» et à peine $2 \%$ « pas assez ». II faut noter que, pour le montant de l'héritage évoqué dans la question, le taux d'imposition de $20 \%$ qui y est proposé est à peu près celui qui est effectivement appliqué par le fisc, au moment de l'enquête. Nul doute donc qu'une majorité de Français trouve ce taux trop élevé et que cette conviction se renforce encore bien davantage lorsqu'il s'agit de transmettre une maison de famille ${ }^{7}$.

7. L'enquête récente du CREDOC (juin 2017, $\mathrm{n}=2004$ ) sur la fiscalité des transmissions patrimoniales (Grégoire-Marchand, 2018) permet également de constater la très forte impopularité de l'impôt sur les héritages ou les donations. Ce résultat vaut quel que soit le niveau de revenu de l'enquêté ou celui de son patrimoine. Les Français se montrent de plus peu favorables à une évolution de la fiscalité actuelle. 


\section{Des attitudes confirmées au sein de l'enquête qualitative sur trois générations}

On retrouve d'ailleurs ce même mouvement dans notre enquête qualitative où ces deux questions ont été posées en des termes proches. 51 enquêtés sur les 94 qui se prononcent, soit $54 \%$, trouvent ce taux « injuste » pour un patrimoine en général et 76 sur 95 , soit $80 \%$, lorsqu'on leur propose de faire l'hypothèse que ce patrimoine se compose uniquement de la maison familiale.

Ainsi, non seulement les inégalités de patrimoine sont mieux tolérées que d'autres inégalités, même en restant dans le champ économique, mais le mécanisme de transmission par héritage n'est pas fondamentalement remis en cause. Cela tient au moins en partie au fait que, pour beaucoup de Français, des parents devraient pouvoir transmettre à leurs enfants leur maison de famille, sans qu'elle se trouve amputée par un prélèvement fiscal significatif.

Cela ne signifie pas que les enquêtés n'aient pas malgré tout conscience de ce que la transmission du patrimoine contribue, d'une manière générale, à la reproduction des inégalités. Dans les entretiens semi-directifs, 60 sur 97 qui ont une opinion, soit $62 \%$, sont de cet avis ; $20 \%$ pensent le contraire et $18 \%$ ne savent pas vraiment. Ainsi David, 46 ans, enseignant en lycée professionnel dans le Béarn, est catégorique : "C'est un mauvais jeu de mots, mais c'est un rôle capital, et pas seulement au niveau de la transmission de l'héritage matériel, immobilier et financier, mais aussi au niveau de l'héritage intellectuel. Les deux vont de pair. " De même, Jean-Marie, 71 ans, retraité de la fonction publique en région parisienne constate que "c'est vrai que ceux qui naissent avec une cuillère en argent dans la bouche, sont effectivement avantagés puisqu'au départ... ils sont dans une situation supérieure aux autres. Donc évidemment ils vont avoir tendance à arriver plus vite plus haut. Donc on peut dire que l'héritage en soi a un effet pervers, qui ne met pas tout le monde sur un pied d'égalité au départ. »

En revanche, Norbert, 68 ans, agent immobilier retraité en région parisienne, récuse ce propos: "Oui, alors ça c'est faux. (...) Les montants de ce qui est transmis diminuent au fil des générations. Et c'est assez classique quand même comme situation. " De même son fils, Sébastien, 45 ans, héritier de l'agence immobilière familiale en région parisienne, considère que les inégalités s'atténuent au fil des transmissions de génération en génération : "À chaque héritage, il est atténué (...) Enfin il n'y a plus de principe aristocratique pur, donc il y a un amortis- 
sement à chaque génération sur les lignées du fait de l'imposition sur les successions etc. (...) Il y a des taxations... à chaque embranchement. Et, oui le patrimoine à l'instant T effectivement il peut être découpé en quatre, en cinq, plus généralement en deux. »

Parmi les $18 \%$ qui ne se prononcent pas vraiment sur la question de la reproduction des inégalités par la transmission du patrimoine, certains ont un point de vue singulier ; comme Nils, 47 ans, cadre en reconversion professionnelle à Paris, qui pense que les inégalités de patrimoine peuvent certes exister mais être compensées par ailleurs: "Pour les très riches, ça se passe bien. Pour les classes moyennes, après c'est vrai bon qu'il peut y avoir des écarts plus ou moins grands. Est-ce que ça influe sur le déséquilibre? Je ne sais pas. Parce qu'entre un cerveau bien fait, et qui n'a pas d'héritage, ou quelqu'un qui a un cerveau très mal fait et qui a un gros héritage, j'ai l'impression qu'on peut compter les points assez facilement. Les deux ont leurs inconvénients. Donc je n'aurais pas un avis très tranché. Je pense que sur le produit intérieur du bonheur, je pense que les deux... auraient leurs problématiques. »

II n'empêche que pour 83 enquêtés sur 103 qui se prononcent, soit $78 \%$, il reste important de transmettre un patrimoine à ses enfants. Si $15 \%$ n'arrivent pas à se former une opinion, ils ne sont que $7 \%$ à être d'un avis contraire. Ainsi, Simone, 95 ans, en maison de retraite du côté d'Aix-en-Provence, qui n'a jamais travaillé de sa vie et qui est issue d'une famille au patrimoine substantiel déclare que transmettre aux enfants: "C'est une continuation de la famille. Et pour certains, la famille c'est très important. Je sais bien qu'il y a des gens pour qui la famille ne compte plus, et qui veulent s'en séparer. Mais pour moi c'est très important. »

La contradiction qu'on pourrait y voir avec la conscience que cela contribue à la reproduction des inégalités n'est qu'apparente car, audelà de l'importance accordée à la transmission patrimoniale, une petite majorité des enquêtés (45 sur 83 qui ont une opinion, soit $54 \%$ ) pense qu'il n'est peut-être pas nécessaire de donner la totalité de son patrimoine à ses enfants afin de leur laisser la latitude de « se faire tout seul » ; $4 \%$ n'ont pas d'avis net tandis qu'une minorité tout de même assez nombreuse (35 sur 83 qui ont un avis, soit $42 \%$ ) pense le contraire. En somme, l'opinion est assez partagée sur cette question. Slimane, 43 ans, commerçant ambulant en fruits et légumes en région parisienne est un fervent défenseur du « se faire tout seul » : « Je pense que c'est important de réussir par soi-même et de voilà quoi (...). Oui enfin je ne trouve pas ça naturel I'héritage. Enfin l'héritage patrimonial en tout 
cas. » En revanche, Vanessa, 45 ans, déléguée syndicale CFDT en Alsace pense que " c'est une connerie. (...) tu peux te passer de l'aide des parents, mais je veux dire, je pense que ça fait partie de ton rôle de parents que d'aider tes enfants. C'est un devoir. "

Quoi qu'il en soit, on ne va pas jusqu'à penser que ce serait une bonne chose que d'avoir la liberté de pouvoir déshériter complètement un de ses enfants. Sur les 78 individus qui ont une opinion à ce sujet, 24 (soit $31 \%$ ) jugent que ce serait une mesure souhaitable, tandis que 52 (soit $67 \%$ ) la réprouveraient ( 2 enquêtés $n$ 'ont pas un avis net et les autres ne se prononcent pas). Parmi ceux qui trouvent que ce serait une mesure souhaitable on trouve des jeunes comme Anna, 19 ans, étudiante à Paris pour qui : "ce qui est à nous, est à nous, donc en soi on le donne à qui on veut, et je ne pense pas qu'il doit y avoir une obligation de donner à quelqu'un si on n'a pas envie. » En revanche, Jean-Pierre, 71 ans, cadre supérieur retraité en région parisienne, se positionne contre cette mesure : "Non moi ça me paraît normal qu'on ne puisse pas déshériter ses enfants. À la limite on peut faire des partages qui peuvent être considérés comme inégaux parce qu'il y a quand même la part réservataire mais en fait bon... On a des enfants il faut bien les assumer. Ça me paraît normal qu'on ne puisse pas les déshériter. »Pour Luce, 83 ans, chanteuse et comédienne retraitée, en région parisienne cela reste vrai même si l'enfant ne le «mérite» pas: "Quoi que cet enfant vous ait fait. Si cet enfant a fait le contraire de ce que vous attendiez de lui, vous a fait souffrir, vous a fait du mal, vous a mis en... Eh bien malgré tout il ne mérite pas d'être déshérité. Au contraire il mérite de... Qu'il sache qu'on est là quand même. Non. Non. On ne peut pas... » Nous allons cependant voir que cette inconditionnalité ne fait pas consensus.

Il est vrai que notre question est exigeante puisqu'il s'agit de déshériter complètement un de ses enfants. Dans un sondage représentatif réalisé en 2014 (CDSP-INED-ELIPSS-SOLIGENE, $\mathrm{n}=770^{8}$ ), deux des questions qui y étaient posées étaient moins contraignantes. On peut se demander dès lors si les résultats vont dans le même sens.

Le premier scénario proposé était le suivant: "Après la mort soudaine de leur père de 90 ans, ses trois enfants apprennent que ce dernier a rédigé un testament qui favorise l'un d'entre eux. II s'agit de sa fille Annie, qui s'est le plus occupée de lui les dernières années de sa

8. Cf. https://cdsp.sciences-po.fr/fr/ressources-en-ligne/ressource/fr.cdsp.elipss.ddi.2014.02. soligene /\#presentation 
vie. Sa sœur, Yvette, et son frère, Pierre, tout en rendant visite fréquemment à leur père, s'en sont beaucoup moins occupés. Yvette et Pierre trouvent le testament injuste. Selon vous, les dispositions testamentaires favorisant Annie sont-elles justes ou injustes? »

\begin{tabular}{lcc}
\multicolumn{1}{c}{ Échelle de réponses } & Nombre & $\%$ \\
\hline 1 - Les dispositions favorisant Annie sont injustes & 52 & 6,8 \\
$--2-$ & 75 & 9,9 \\
$--3-$ & 206 & 27,1 \\
$--4-$ & 269 & 35,4 \\
5 - Les dispositions favorisant Annie sont justes & 158 & 20,8 \\
Ne sait pas & 10 & - \\
\hline
\end{tabular}

Une majorité relativement importante (56\%) pense que le " mérite » d'un des enfants relativement aux autres peut légitimement conduire à le favoriser au moment de la succession. Dans notre enquête qualitative, Pierrelise, 54 ans, ancienne employée de la distribution alimentaire, femme d'artisan-maçon, désormais inactive, du côté de Toulon le formule ainsi : "Oui ça me paraît juste. Oui parce qu'il y a quand même des enfants qui sont suffisamment ingrats et méchants avec leurs parents, pour... C'est quand même une récompense quelque part. Et le travail des parents d'avoir épargné de l'argent, et quand même que ce soit ceux qui leur ont rendu en amour, et en affection, en tendresse. Je ne sais pas. C'est normal de privilégier un des enfants, s'il a été plus présent, qu'il a donné plus d'amour. »

En revanche, face au second scénario proposé («Madame Denave est sollicitée par une association humanitaire pour faire une donation. En froid avec ses deux enfants, elle envisage de faire un legs important à l'association. Selon vous, est-ce juste ou injuste envers ses enfants? »), les enquêtés sont plus dubitatifs. Donner moins à ses enfants pour faire un don important à une association humanitaire n'emporte pas la conviction. Ceux qui trouvent cela injuste sont plus nombreux (37\%) que ceux (24\%) qui pensent le contraire.

On retrouve donc cette idée majoritaire dans notre enquête qualitative qu'il n'est pas souhaitable de pouvoir déshériter ses enfants mais, comme on le voit à présent, même pour une cause humanitaire. Seul le dévouement auprès de(s) parent(s) peut amener à accepter l'idée que soit favorisé l'enfant dévoué, au détriment des autres, et donc au bout du compte à accepter l'idée de déshériter partiellement ces derniers. 


\begin{tabular}{lcc}
\multicolumn{1}{c}{ Échelle de réponses } & Nombre & $\%$ \\
$\mathbf{1}$ - Cette donation à I'association est injuste & 127 & 16,7 \\
$--2-$ & 155 & 20,4 \\
$--3--$ & 292 & 38,5 \\
$--4-$ & 120 & 15,8 \\
5 - Cette donation à l'association est juste & 65 & 8,6 \\
Ne sait pas & 11 & - \\
\hline
\end{tabular}

Toutes ces données, et les hésitations qui les accompagnent parfois, semblent confirmer que les enquêtés opèrent des distinctions à l'intérieur de l'héritage patrimonial en acceptant certaines transmissions et en refusant d'autres. Par exemple, comme nous l'avons vu, ils valident la transmission de la maison familiale (sans amputation fiscale forte) mais sont plus dubitatifs pour ce qui est du reste. Pour savoir comment leurs discours s'organisent, voyons plus précisément comment se décline leur argumentation.

\section{Trois types de discours à propos de la transmission patrimoniale}

À cette fin, nous avons effectué une analyse factorielle à titre exploratoire et deux dimensions ont semblé structurantes. D'une part, l'importance accordée à la transmission patrimoniale, ainsi que le rôle reconnu à cette transmission dans la reproduction sociale. Et, d'autre part, l'opinion sur l'idée de «se faire tout seul». Compte tenu des effectifs, il en ressort trois types de discours. Tout d'abord, un petit groupe d'enquêtés se distinguent, en jugeant qu'il n'est pas si important de transmettre un patrimoine à ses enfants. Ensuite, parmi les autres enquêtés, une partie défend clairement les solidarités familiales et se montre un peu plus sceptique face aux critiques qui y voient une source de reproduction sociale et face à l'idée de « se faire tout seul ». Enfin, le troisième groupe insiste pour sa part sur la liberté de se constituer un patrimoine et de le transmettre librement, notamment à sa progéniture.

Dans une certaine mesure, et quoique imparfaitement, ces trois discours ne sont pas sans rappeler la typologie proposée par André Masson sur les différentes conceptions de l'individu lorsqu'il est question du lien intergénérationnel (Masson, 2009). Pour Masson, la solidarité intergénérationnelle est pensée à partir des trois piliers que sont le marché, la famille et l'État. Selon la manière de hiérarchiser l'importance respective de ces piliers, il repérait trois types de pensée, 
chacune accordant sa confiance à un des piliers tout en se défiant d'un des autres. Il distinguait ainsi :

- La pensée «libérale" ou du libre agent, qui s'appuie sur le marché et se méfie de l'État. L'individu est responsable de son bien-être et doit être libre de ses choix. II doit pouvoir transmettre ce qu'il a accumulé comme il l'entend.

- La pensée « conservatrice » ou multisolidaire, qui s'appuie sur la famille et se méfie du marché. Les individus sont définis par des liens de fraternité plus ou moins étroits (de ceux familiaux à ceux nationaux, notamment) qui justifient des solidarités plus ou moins fortes.

- La pensée «sociale-démocrate » ou de l'égalité citoyenne, qui mise d'abord sur l'État pour mettre tous les individus à égalité et, en revanche, se méfie des liens familiaux, considérés comme la matrice des traitements partiaux.

Dans le cas présent, nous distinguerions le discours du libre agent qui défend sa propriété et son droit de la transmettre, le discours familialiste soucieux de bien transmettre son patrimoine au sein de sa lignée et, enfin, le discours qui relativise l'importance de la transmission patrimoniale, au nom de l'égalité de départ dans la vie ou pour d'autres raisons.

Nous avons classé les entretiens en fonction des réponses à deux questions. Il s'agissait de savoir, d'une part, s'il était important de transmettre un patrimoine à ses enfants et, d'autre part, de savoir s'il ne fallait pas forcément tout transmettre à ses enfants dans l'idée qu'ils se fassent tout seul. Toutefois, il n'a pas été possible de classer tous les entretiens selon ces deux critères, la réponse à l'une ou l'autre des deux questions étant soit ambivalente, soit manquante. Sur les 105 entretiens, nous en avons donc retenus 70 dont les réponses étaient suffisamment tranchées.

Parmi ceux-ci, c'est le discours du libre agent qui est le discours le plus fréquent (34 entretiens). Il s'agit du discours des plus jeunes générations (16 des 34 enquêtés), qui se lancent dans la vie et insistent sur l'importance de se faire tout seul. Ils sont bien conscients que l'héritage favorise la reproduction sociale (22 enquêtés l'affirment, contre 6 qui disent l'inverse), néanmoins ils jugent plus essentiel de pouvoir transmettre ce qui a été acquis par leur travail. Ils défendent tout d'abord la liberté de pouvoir disposer comme ils l'entendent de ce qu'ils ont 
acquis, quitte à déshériter un enfant qui aurait démérité. Mais, les enfants demeurent, à leurs yeux, les héritiers privilégiés.

Ainsi Thibaut, 20 ans étudiant en STAPS à Montpellier, dit à propos de l'individu en position de transmettre un patrimoine: "C'est ses biens à lui. Ce n'est pas à la loi de décider ce qu'il doit faire de son argent, et de ses biens (...) Ce n'est pas l'État qui a fait en sorte qu'il ait gagné tout ça. Toute sa vie, cet homme-là, il a donné déjà des impôts par rapport à ce qu'il avait (...) Au fur et à mesure qu'il gagnait de l'argent, il donnait de plus en plus. Donc je trouve ça encore plus bizarre qu'après sa mort, il ne puisse même pas en plus profiter de tout ce qu'il a gagné toute sa vie, et de le donner à qui il veut donc... Non pour moi ce n'est pas normal (...) Quelqu'un qui a gagné un truc, c'est pas à l'État de le recevoir, c'est à ses descendants forcément puisque sinon ça lui servait à rien de le gagner. II I'a pas gagné pour l'État. » Puis il ajoute à propos de la personne héritière : "Si à la fin de sa vie, elle l'a encore, bah c'est qu'elle a mérité de le garder donc... Puisque elle a réussi ... à ne pas le perdre. »

Ensuite, le discours familialiste, multisolidaire est également fréquent (29 entretiens). II s'agit notamment du discours des anciennes générations (15 sur 28 enquêtés), qui songent à transmettre à leurs enfants. Pour ces enquêtés, les solidarités familiales priment sur le reste. Aussi la transmission patrimoniale ne pèse pas tant que cela sur la reproduction sociale. Le patrimoine $n^{\prime}$ est pas simplement une valeur financière, mais il est aussi porteur d'une histoire familiale, de moments partagés, et s'il ne l'est pas de facto, il devrait l'être. La famille idéale se retrouve ainsi autour d'une maison familiale pour les vacances, et chaque génération y a ses propres souvenirs. Chacun vient y puiser des racines, s'y ressourcer, avant de repartir dans ses projets individuels. En ce sens, il n'est pas question de penser que l'on se ferait tout seul, en aliénant une partie du patrimoine familial. C'est au contraire en s'appuyant sur lui que l'on trouve la force d'agir.

Cela est illustré par les propos de Bernadette, 74 ans, cadre supérieure retraitée en région parisienne qui nous dit : "Moi je pense... à I'héritage culturel, et je pense que ça c'est un devoir de transmission. Et un devoir de transmission enfin pas forcément... Ça dépend ce que vous appelez héritage (...) Bon qu'il y ait... une solidarité intergénérationnelle... dans une famille, je crois que c'est important. II me semble que la vie devient de plus en plus dure, et que... les jeunes... On ne peut pas ne pas être attentif à ce qu'ils vivent en fait, et à ce qu'ils risquent. (...) Mais pour moi je vous dis, le patrimoine, ce n'est pas uniquement le patrimoine 
financier. (...) Parce que je pense qu'on est... Je crois beaucoup à la transmission. À l'importance. Et je pense qu'on est... (Silence)... On est dépositaire de quelque chose qui ne nous appartient pas forcément uniquement... Et qu'on a à transmettre aux générations suivantes. »

Enfin, le discours de l'égalité citoyenne est plutôt rare, et ce d'autant plus que sur les 7 entretiens qui relativisent l'importance de la transmission patrimoniale, tous ne mettent pas en avant le souci de l'égalité. Une lignée de trois enquêtés défend avec conviction l'égalité citoyenne contre la transmission. II s'agit d'une famille politiquement très à gauche, plutôt proche du Parti communiste. Pour les autres, la transmission patrimoniale n'apparaît pas véritablement comme une question qui se pose.

Ainsi, Robert, 70 ans, retraité de la SNCF, proche du Parti communiste, en région parisienne, dit à propos de la transmission de patrimoine que c'est "un des premiers facteurs qui reproduit les inégalités. Enfin bon ça moi c'est... Là-dessus je suis assez catégorique. C'est un des premiers facteurs (...). Moi je serais pour la suppression de l'héritage carrément (...). C'est vrai que la France, c'est quand même un pays hyper... inégalitaire, parce que bon pour certains $500.000 € c^{\prime} e s t .$. . C'est le Pérou, c'est la fortune. Et puis pour d'autres c'est... C'est quoi ? C'est... de l'argent de poche sur... peut-être pas sur un mois, mais sur une année. (...) Ah moi les plus riches, je suis d'accord pour les saigner (rires)! " La fille de Robert, Sophie, 44 ans, cadre supérieure du privé, en région parisienne, également proche du Parti communiste ne dit pas autre chose : "Je suis contre l'héritage. (...) Il est évident que I'héritage participe de la reproduction des inégalités. »

\section{Des principes partagés à leurs applications}

II n'est toutefois pas toujours facile de démêler les différents types de discours sur la transmission patrimoniale, notamment entre celui du libre agent et celui familialiste. Les enquêtés mobilisent fréquemment des considérations dans les deux, voire les trois registres. Dans 70 cas, nous avons jugé qu'un type de discours dominait les autres, mais dans 29 cas, aucun ne se démarquait nettement, laissant le propos ambivalent (dans les 6 cas restants par rapport au total des 105, le propos a été jugé difficilement exploitable).

Ceci étant, plutôt que de parler d'ambivalence, nous formerions plutôt l'hypothèse qu'il existe un relatif consensus sur les principes qui 
devraient régir la transmission. En effet, les enquêtés sont sensibles aux trois argumentations typiques que nous avons relevées, celle du libre agent qui d'une certaine manière refuse de payer l'impôt une « deuxième » fois, celle du familialisme qui défend les biens de la famille censés passer d'une génération à la suivante et, enfin, celle de l'égalitarisme qui souligne l'inégalité des chances induite par la transmission patrimoniale. Chacune de ces lignes $d^{\prime}$ argumentation ne pèse certes pas du même poids d'un enquêté à l'autre, mais personne ne s'est opposé frontalement à l'un ou l'autre de ces arguments. Manifestement, chacun de ces principes est jugé valide et non contradictoire avec les deux autres. Comment est-ce possible?

Vraisemblablement, les enquêtés considèrent implicitement que chaque principe s'applique à une certaine forme de patrimoine. Tout d'abord, il y a l'argent que l'on épargne, celui qui est le fruit du travail $d^{\prime}$ 'une vie - c'est le domaine où le discours du libre-agent se montre le plus pertinent. Puis il y a le bien de famille, celui qui porte l'inscription de la vie de famille et de la succession des générations en un même lieu - c'est le domaine où le discours familialiste porte. Enfin, il y a tout le reste, ce qui excède les domaines précédents et sort donc des domaines « préservés » - et là, le discours égalitariste s'applique.

Dans l'esprit des enquêtés, ces formes de patrimoines correspondent à des montants de patrimoines différents. Et de ce point de vue au moins deux niveaux sont distingués. Les principes du libre agent et du familialisme renvoient à des montants de patrimoines ordinaires, aux yeux de l'enquêté, et conduisent à la même conclusion que le fisc doit rester à distance. Le principe égalitariste s'appliquerait lui pour les montants de patrimoine jugés extraordinaires.

Cela est illustré par les propos de Jean-Marie, 71 ans, retraité de la fonction publique en région parisienne : «Est-ce que c'est important de transmettre un patrimoine? Je ne sais pas. Un petit peu. Enfin de toute façon il y a aussi l'idée de laisser quelque chose, une trace. Donc... Que les enfants aient un signe du passage de leurs parents et de leurs grandsparents, ce n'est pas mauvais. Simplement (...) ce qu'il faut trouver c'est : à quel niveau il faut s'arrêter? Et ça, je n'ai pas de réponse définitive. " D'une certaine manière c'est aussi ce que dit Joséphine, 16 ans, lycéenne en région parisienne, puisque sans être absolument contre I'héritage, elle déclare: "Quand il est trop important, je serais pour qu'il $y$ ait une taxe dessus. Enfin pour ne pas qu'il soit trop, trop important non plus parce que oui c'est un héritage, mais c'est assez injuste de vivre de 
rentes toute sa vie... pendant que d'autres gens travaillent toute la journée, et ne gagnent même pas un quart de... des rentes. »

Nous ferions donc l'hypothèse que le désaccord porte plus, en matière de succession, sur les niveaux à partir desquels les principes changent, que sur les principes mêmes et leur étagement.

\section{Une importance transversale de la transmission culturelle}

Il faut toutefois remarquer que d'autres aspects de la transmission sont apparus au cours des entretiens. Ainsi, plusieurs enquêtés ont souligné que l'essentiel de la transmission réside dans sa dimension «culturelle», dans le « roman familial » et la transmission de valeurs ce que d'ailleurs d'autres études avaient déjà souligné (Gaulejac, 1999 ; Coenen-Huther, 2000 ; Muxel, 2007). D'autres ont remarqué que I'héritage arrivait aujourd'hui de plus en plus tard et que, finalement, la position sociale se jouait surtout au niveau de cette transmission «culturelle», qui inclut manifestement l'éducation diplômante.

Vanessa, 45 ans, déléguée syndicale CFDT en Alsace, qui dit ellemême avoir manqué de présence parentale à l'adolescence, et qui, comme on l'a vu plus haut, n'adhère pas du tout au principe de se «faire tout seul », note cependant que, l'héritage arrivant tardivement, il n'est peut-être pas si important de transmettre un patrimoine à ses enfants: "Aider au mieux ses enfants, ce n'est pas forcément attendre notre décès pour leur donner un bout de pierre. C'est peut-être les accompagner... dans les études, les accompagner dans la vie quoi (...). C'est ça qui est important. Partir sur de bonnes bases, je pense que c'est à un moment donné les aider au début de leur vie."

Cette importance de la transmission «culturelle » et /ou « affective » apparaît ici transversale aux trois types de discours que nous avons distingués.

Ainsi la transmission «culturelle » est jugée importante pour les libres-agents qui trouvent que l'État n'a pas à s'immiscer dans la transmission familiale. Dans cette représentation, chacun fait ce qu'il veut de son argent et de son patrimoine, le donne à ses enfants comme il veut, sans que l'État n'ait à intervenir. La transmission est alors perçue comme une affaire personnelle et affective. Pour les familialistes, transmettre un patrimoine, c'est inscrire les enfants dans la lignée d'une histoire familiale, au-delà du patrimoine financier, quitte à ce que l'État 
rétablisse les déséquilibres pour que la transmission ne pèse pas sur la reproduction sociale. Quant aux citoyens égalitaristes, ils trouvent normal d'avoir envie de laisser quelque chose à ses enfants parce que c'est «naturel », même si cela doit rester dans des proportions raisonnables et en taxant au maximum les plus grosses transmissions ( il faut saigner les riches ») pour tendre vers l'égalité. Enfin pour les ambivalents - position intermédiaire entre celle des libres-agents et des familialistes -, qui pensent que la transmission à l'intérieur de la famille est naturelle, la transmission culturelle est plus importante que la transmission patrimoniale (au sens purement financier du terme). Ce groupe va jusqu'à considérer que la transmission patrimoniale contient la transmission «culturelle/affective ».

Notre typologie ne recouvre pas totalement celle obtenue par Anne Gotman à partir de son enquête par entretiens semi-directifs (Gotman, 1988 ) réalisée en 1985-1986 auprès de 50 salariés résidant en milieu urbain et venant de faire un héritage. La méthodologie utilisée n'est toutefois pas la même que la nôtre et rend difficile la comparaison des résultats. Son enquête se centre sur des pratiques à l'égard d'héritages bien précis et récents et non, comme dans notre cas, sur des opinions à propos de l'héritage en général. On observe cependant des similitudes dans certaines argumentations.

On retrouve par exemple chez ceux que Gotman appelle les « éducateurs » l'argument que nous venons de mettre en évidence quant à l'importance de la transmission culturelle. II apparaît notamment qu'à partir du moment où les héritages économiques ne pèsent plus autant sur les destins sociaux, les inégalités de patrimoine ne sont plus aussi inacceptables. On retrouve par ailleurs, en particulier chez ceux que Gotman nomme les «fondateurs », les arguments défendus par nos « familialistes».

\section{Un effet de la lignée}

Au-delà de notre typologie et malgré la petite taille de l'échantillon, notre enquête permet également de repérer la proximité des opinions entre membres d'une même lignée. Il est assez remarquable d'observer de tels effets avec seulement 34 lignées observées (la $35^{\mathrm{e}}$ ne comportant qu'un individu n'est pas pertinente ici). Quels sont ces effets?

Tout d'abord, la famille joue manifestement un rôle dans la socialisation politique. Le résultat est bien connu (Percheron, 1997) et il est 
confirmé ici. Un test non paramétrique de Kruskal-Wallis du positionnement politique selon les lignées fait ressortir significativement les lignées de droite et les lignées de gauche $(p=0,4 \%)$. II n'est bien sûr pas possible de démêler les différents canaux, directs et indirects, de cette transmission familiale du positionnement politique, qui peut traduire à la fois une transmission de valeurs, une reproduction du positionnement social, du niveau de diplôme, du niveau de revenu, voire une défense conjointe du patrimoine familial, etc.

Une distinction statistiquement significative $(p=1,5 \%$ au test de Kruskal-Wallis) apparaît également entre les lignées qui jugent important de transmettre un patrimoine et celles qui jugent cela peu ou pas important. Ceci pourrait peut-être s'expliquer par le fait que certaines lignées ont un patrimoine à transmettre et les autres, non; mais nous ne pouvons pas le vérifier ici précisément. Enfin, on repère également un écart significatif ( $p=5,8 \%$ au test de Kruskal-Wallis) entre les lignées qui trouvent juste de payer un impôt de 20 \% sur les successions de capitaux et celle qui trouvent cet impôt excessif.

D'autres résultats sont encore perceptibles malgré la petite taille de l'échantillon. Ils éclairent assez bien les principaux ressorts de l'opinion concernant le patrimoine et l'héritage. Les individus les plus jeunes insistent sur la nécessité de "se faire tout seul », tandis que les anciens soulignent l'importance de transmettre autant que possible. Les premiers déclarent également moins souvent que transmettre un patrimoine est important, alors que ce souci se renforce nettement avec l'âge.

On observe également que les lignées de droite relativisent plus fortement le rôle de la transmission patrimoniale sur la reproduction sociale que les lignées de gauche. Et, enfin, qu'elles s'opposent plus à l'imposition sur les successions, qu'il s'agisse de la transmission d'un capital ou d'une maison.

\section{Conclusion}

Les travaux que nous avons ici abordés, qu'ils soient de nature statistique ou issus d'entretiens semi-directifs, attestent d'une tolérance assez forte, en France, aux inégalités de patrimoine, eu égard à d'autres formes d'inégalités, ainsi que d'une adhésion au principe de transmission par héritage. Pourquoi ? Deux ordres de raison se sont nettement détachés. D’un côté, certains enquêtés ont défendu leur liberté de 
transmettre comme ils le souhaitent les biens leur appartenant, et ce dans une veine très smithienne. Selon eux, ce qu'ils ont gagné ne doit pas leur être repris à leur mort. Sans être totalement hostiles à une taxe sur les successions, ils considèrent que celle-ci doit être particulièrement limitée. D'un autre côté, d'autres enquêtés ont mis en avant l'importance que revêt pour eux la lignée familiale, en insistant sur ce que les ascendants doivent à leurs descendants, en défendant la réciprocité familiale, générationnelle, où idéalement il faut « rendre » à ses enfants ce que l'on a reçu de ses parents. Au total, nombre de Français souhaiteraient pouvoir transmettre à leurs enfants une maison de famille - archétype du bien « lieu de mémoire » d'une histoire familiale - et ne pas être acculés à sa vente, en raison d'une fiscalité qui serait perçue comme confiscatoire. II faut d'ailleurs noter que sur ces questions les membres d'une même lignée ont tendance à partager des opinions proches.

Mais un troisième ordre de raison est également apparu. En vertu d'un idéal d'égalité citoyenne, certains enquêtés se sont montrés soucieux de raboter nettement l'héritage dans une perspective rousseauiste ou même, pourrait-on dire aussi, durkheimienne (Durkheim, 1950). Ce dernier discours tient nettement moins de place dans les entretiens que les deux précédents. Pour autant, nous n'avons pas rencontré d'opposition frontale à cette revendication égalitaire. Au contraire, les enquêtés sont bien conscients que l'héritage constitue une entrave à l'égalité des chances, qu'ils défendent par ailleurs. Pourtant, à leurs yeux, et aussi surprenant cela soit-il, il n'y a pas vraiment de contradiction. Pour les niveaux de patrimoine auxquels ils songent spontanément, des niveaux « ordinaires » qui s'avèrent assez peu élevés, ils manifestent une très forte aversion face à l'idée de taxation sur la succession. Celle-ci doit être faible. Sinon elle est rapidement perçue comme "confiscatoire ». S'il y a une base pour justifier une taxation sur l'héritage, celle-ci est clairement limitée, dominée même, par les raisons du libre agent et du familialisme. Cette forte résistance à la taxation ne concerne toutefois pas les niveaux de patrimoine jugés extraordinaires, sur lesquels une taxation justifiée par l'égalité citoyenne n'est cette fois guère contestée.

Ceci dit, de nombreux enquêtés ont fait remarquer que l'héritage patrimonial arrivait assez tard au cours de la vie de l'héritier et que $d^{\prime}$ autres formes de transmissions, d'autres aides, s'avéreraient finalement plus importantes, en intervenant plus précocement. Cela 
plaiderait pour des mesures favorisant les donations. Mais au-delà, faudrait-il y voir des arguments en faveur d'un capital conféré à tous au début de la vie adulte (Huet, 1853) ? C'est ce que des travaux ultérieurs pourront dire.

\section{Références}

Argouarc'h J., Boiron A., 2016, «Les niveaux de vie en 2014 », Insee Première, $\mathrm{n}^{\circ} 1614$.

Arrondel L., 2009, « Mon père avait raison : la transmission des valeurs entre les générations ", Revue Française d'Économie, 2009, XXIV, n 1 , p. 157-205.

Coenen-Huther J., 2000, La mémoire familiale, Paris, L'Harmattan.

Durkheim E, 1950, Leçons de sociologie, Physique des mours et du droit, Istanbul, Cituri Biraderler Basimevi; $4^{\mathrm{e}}$ éd, Paris, Puf, coll. "Quadrige », 2015.

Ferrante A., Guillas D., Solotareff R., 2016, « Entre 2010 et 2015, les inégalités de patrimoine se réduisent légèrement », Insee Première, $\mathrm{n}^{\circ} 1621$.

Forsé M., 2013, «Une passion de l'égalité particulièrement insatiable en France », Revue de l'OFCE, $131: 5-18$.

Forsé M., Galland O. (sous la dir de), 2011, Les Français face aux inégalités et à la justice sociale, Paris, Armand Colin.

Forsé M., Galland O., Guibet Lafaye C., Parodi M., 2013, L'égalité, une passion française?, Paris, Armand Colin.

Forsé M., Parodi M., 2015, "Les Français et la justice fiscale », Revue de l'OFCE, $137: 97-132$.

Galland O. (sous la dir. de), 2016, La France des inégalités, Réalités et perceptions, Paris, Presses de l'Université Paris Sorbonne.

Gaulejac V. de, 1999, L'histoire en héritage, Roman familial et trajectoire sociale, Paris, Desclée de Brouwer ; rééd. Paris, Payot, 2012.

Gotman A., 1988, Hériter, Paris, P.U.F.

Grégoire-Marchand P., 2018, «Fiscalité des héritages : connaissances et opinions des Français », France Stratégie, Document de travail 2018-2.

Huet F., 1853, Le Règne Social du Christianisme, Paris/Bruxelles, Firmin Didot Frères/Librairie Polytechnique Decq.

Masson A., 2009, Des liens et transferts entre générations, Paris Éditions de l'EHESS.

Muxel A., 2007, Individu et mémoire familiale, Paris, Hachette. 
Percheron A., 1993, La socialisation politique, textes réunis par Mayer N. et Muxel A., Paris, Armand Colin.

Percheron A., 1997, La socialisation politique, Paris, Armand Colin.

Percheron, A., 1982, "Préférences idéologiques et morale quotidienne d'une génération à l'autre ", Revue française de science politique, 32 (2) ;185-209.

Rousseau J.-J., Discours sur l'économie politique, 1755, in Cuvres complètes, t. III, Paris, 1964.

Smith A., Leçons sur la jurisprudence, 1762-1764, Paris, Dalloz, 2007. 